\title{
BMJ Open Comparison of self-reported measures of alcohol-related dependence among young Swiss men: a study protocol for a cross-sectional controlled sample
}

\author{
Katia Iglesias, ${ }^{1,2}$ Frank Sporkert, ${ }^{3}$ Jean-Bernard Daeppen, ${ }^{4}$ Gerhard Gmel, $, 4,6$ \\ Stephanie Baggio ${ }^{7,8}$
}

To cite: Iglesias $\mathrm{K}$,

Sporkert F, Daeppen J-B, et al. Comparison of selfreported measures of alcoholrelated dependence among young Swiss men: a study protocol for a cross-sectional controlled sample. BMJ Open 2018;8:e023632. doi:10.1136/ bmjopen-2018-023632

- Prepublication history for this paper is available online. To view these files, please visit the journal online (http://dx.doi. org/10.1136/bmjopen-2018023632).

Received 20 April 2018 Revised 5 June 2018 Accepted 6 June 2018

Check for updates

(C) Author(s) (or their employer(s)) 2018. Re-use permitted under CC BY-NC. No commercial re-use. See rights and permissions. Published by BMJ.

For numbered affiliations see end of article.

Correspondence to

Dr Katia Iglesias;

katia.iglesias@hefr.ch

\section{ABSTRACT}

Introduction Short screenings of alcohol-related dependence are needed for population-based assessments. A clinical interview constitutes a reliable diagnosis often seen as gold standard, but it is costly and time consuming and as such, not suitable for population-based assessments. Therefore, self-reported questionnaires are needed (eg, alcohol use disorder (AUD) as in the Diagnostic and Statistic Manual of Mental Disorders (DSM) 5), but their reliability is questionable. Recent studies called for more evidence-based measurements for population-based screening (eg, heavy alcohol use over time (HAU)). This study aims to test the reliability of different self-reported measures of alcohol use.

Methods and analysis Based on stratified random selection, 280 participants will be recruited from the French-speaking subgroup of the Swiss National Science Foundation-supported Cohort Study on Substance Use and Risk Factors (C-SURF). This cohort is a population-based sample of young Swiss men in their mid-20s $(n=2668)$. The sample size calculation is based on a proportion non-inferiority test (alpha $=5 \%$, power $=80 \%$, margin of equivalence $=10 \%$, difference in sensitivity between selfreported $\mathrm{AUD}$ and $\mathrm{HAU}=5 \%$, correlation between $\mathrm{AUD}$ and $\mathrm{HAU}=0.35$, and drop-outs=15\%). Assessment will include a clinical interview as the gold standard of alcohol-related dependence, self-reported alcohol measures (HAU, AUD and drinking patterns), biomarkers as gold standards of chronic excessive drinking, and health outcomes. To assess the validity of the self-reported alcohol measures, sensitivity analyses will be run. The associations between alcohol-related measures and health outcomes will be tested. A non-response analysis will be run using the previous waves of the C-SURF study using logistic regressions.

Ethics and dissemination The study protocol has been approved by the Human Research Ethics Committee of the Canton of Vaud, Switzerland (no. 2017-00776). The results will be submitted for publication in peer-reviewed journals and presented at national and international conferences.

\section{BACKGROUND}

Substance-related dependence is a major health concern worldwide, with alcohol
Strengths and limitations of this study

- Evaluation of self-reported outcomes compared with a clinical interview based on the DSM- 5 .

- Inclusion of a large number of outcomes: clinical interview, biological material, and self-reported measures.

- Nested project in a longitudinal study: Iongitudinal data available for the participants included in the sample, non-response analysis.

Only men in their mid-20s.

- The available data to test reliability of the self-reported measures are separated by one year.

being described as the substance leading to the most disabling mental disorders. ${ }^{1}$ Defining and measuring substance-related dependence is difficult and has led to various changes according to social, economic and political reasons. ${ }^{2}$ Indeed, substance-related dependence went through several shifts in terminology, definition, and measurement over the last 50 years. ${ }^{23}$ These changes were designed to improve its measure and aimed to be scientifically valid, clinically useful and understandable by the general public. ${ }^{4}$ Generally speaking, there is an agreement to define substance-related dependence as a syndrome of physiological, behavioural and cognitive phenomena developed after repeated substance use. ${ }^{5-7}$ Therefore, 'alcohol-related dependence' can be defined as a syndrome of physiological, behavioural and cognitive phenomena developed after repeated alcohol use. We prefer this term instead of 'alcohol dependence', which would be misleading because alcohol dependence has been used to define a distinct disorder in, for example, in the Diagnostic and Statistic Manual of Mental Disorders (DSM) $\mathrm{IV}^{7}$ and International Classification of Diseases (ICD) 11 and no longer exists in the DSM-5, which 
combines two disorders, abuse and dependence, into alcohol use disorder (AUD).

\section{Measuring alcohol-related dependence: the gold standard}

Assessing alcohol-related dependence requires a clinical interview conducted by an experienced clinician in direct exchange with a patient. Indeed, a clinical interview provides a reliable diagnosis and it is often seen as a gold standard. Without an extensive anamnesis, it is difficult to establish a reliable diagnosis because alcohol-related dependence is a syndrome with several physiological, behavioural, cognitive and psychological processes, and not just 'a tick box of symptoms'.

Beyond clinical interviews, biochemical investigations are also used to assess chronic excessive drinking ${ }^{9}$ without asking people about their alcohol use. Biomarkers do not allow direct testing of the concept of alcohol-related dependence. However, they may be useful to screen for chronic excessive drinking, which may be a strong indicator of alcohol-related dependence. Since they do not rely on self-reports nor judgements of a clinician, they are of great interest in alcohol research.

However, clinical diagnoses and biomarker analyses are costly and time consuming and therefore not suitable for general population assessments that are needed for public health planning and monitoring, such as establishing prevalence rates, treatment planning, policy-making, and early intervention. Therefore, short quantitative measures of alcohol-related dependence are needed.

\section{Alcohol-related dependence self-reported measures}

Several self-reported measures of alcohol-related dependence are already available. In the recent developments of the DSM-5, alcohol-related dependence is measured through eleven criteria designed to diagnose AUD. ${ }^{10}$ However, despite the fact that AUD is well defined and that its measure addresses previous issues related to the diagnosis of the DSM-IV, ${ }^{11}$ several studies reported difficulties related to alcohol-related dependence's measurement using self-reported measures (eg, refs 12-14). For example, the self-reported questions based on the criteria of AUD ${ }^{10}$ may be misinterpreted by respondents. Previous studies highlighted misinterpretation of DSM diagnostic criteria, ${ }^{1315}$ contamination by negative thinking patterns of depressive people, ${ }^{16}$ lack of specificity, ${ }^{14}$ low positive predictive values (meaning that those who screen positive do not have the disorder), ${ }^{17}$ and lack of convergence with clinical diagnoses. ${ }^{5}$ Young heavy drinkers are especially concerned. They are likely to misinterpret survey questions and to share a misperception of AUD symptoms, such as after-effects and acute intoxication. Therefore, they are likely to over-report physiological symptoms of withdrawal and tolerance. ${ }^{12}$ Moreover, it seems that self-reports are not always consistent with clinical diagnoses. However, misspecification of self-reported AUD is understudied. ${ }^{12}$

As a consequence of these pitfalls, a recent study called for more evidence-based measures. ${ }^{2}$ Some previous studies proposed heavy use as a suitable criterion in future classifications of substance-related dependence. ${ }^{2} 1819$ Rehm $e t a l^{2}$ suggested that alcohol use over time, and more specifically heavy alcohol use (HAU) over time, is responsible for the physiological changes, symptoms, social consequences and burden of disease associated with the current definition of alcohol-related dependence. They concluded that HAU should be a relevant indicator of alcohol-related dependence. Moreover, the use of HAU also may diminish stigmatisation associated with alcohol-related dependence ${ }^{5} 182021$ since alcohol use over time is less stigmatised than AUD. However, there are at least two important issues. The first one is the lack of definition of HAU: how many drinks are needed to defined 'heavy use', and how many months are needed to define 'over time'? Currently, some indicators of alcohol use over time are available; for example, two drinks per day maximum is defined as low-risk alcohol consumption. ${ }^{22}$ Second, some studies reported that HAU is not a sufficient indicator of addictive behaviour, ${ }^{23}$ but empirical studies investigating this question using reliable measures of alcohol-related dependence have not been conducted. This measure does not aim to replace clinical assessments, which are compulsory for diagnostic evaluation and treatment, but would be of great interest for general population screening purposes. Thus, there is still a lack of consensus on which measure should be used and of empirical studies designed to test its reliability.

An alternative operationalisation of alcohol-related dependence has recently been suggested by Martin $e t a l^{24}$ They proposed that substance use disorders should focus on what they called 'core' features (ie, primary symptoms indexing internal dysfunctions) and not on 'ancillary' features (ie, consequences). According to these authors, consequences should not be used to measure substance-related dependence because they are context dependent, manifoldly determined and not necessarily related to one substance but to multiple substances. It is well established that AUD is associated with several detrimental consequences as consequences are part of the DSM- 5 definition. However, non-disordered AUD can also result in consequences. ${ }^{14}$ Therefore, Martin et $a l^{24}$ suggested assessing alcohol-related dependence with primary symptoms and removing consequences from its measure in order to get a more reliable measure; for example, to decrease the number of false negatives. To our knowledge, no empirical study tested this proposition, and data are thus needed.

\section{Aim of the study}

Based on clinical interviews designed to diagnose alcohol-related dependence, the main aim of this study is to test the quality of self-reported AUD to assess alcohol-related dependence in the general population. Another aim of this study is to test whether self-reported HAU can be used instead of self-reported AUD as a measure of alcohol-related dependence in a general population-based sample. It will also test whether self-reported 
AUD focusing on primary symptoms and excluding alcohol-related consequences is a better assessment of alcohol-related dependence than self-reported AUD in its traditional definition.

\section{METHODS/DESIGN \\ Study design}

The study is a single-centre, national, controlled study with a stratified random sample selection and a cross-sectional design.

\section{Setting}

The study will be conducted in the Lausanne University Hospital (CHUV) in the Alcohol Treatment Centre. This facility is an urban public hospital serving 770000 people. It is one of the five teaching university hospitals located in Switzerland.

\section{Population and sample \\ Population}

Our study is a large nested project of the ongoing longitudinal Cohort Study on Substance Use and Risk Factors (C-SURF) study supported by the Swiss National Foundation (SNF grant 33CSC0_122679, 33CSC0_139467 and 33CS30_148493). ${ }^{25}$ The C-SURF study is representative of young men around 20 years old. Young men are the study focus because they are a high-risk population regarding alcohol use. ${ }^{26}$ In collaboration with the C-SURF study, participation in the present project will be proposed to all French-speaking participants who were recruited within the Lausanne army recruitment centre and who answer the second follow-up of C-SURF in the following six months with a valid email address $(n=2668)$. French speakers are the targets of this study because C-SURF covered all French speakers, whereas the German-speaking part uses only a subgroup of all German-speaking Swiss men. To focus on French speakers also reduces costs by using only one language for clinical assessment and a narrower area from which people have to travel for the clinical interview. In addition, C-SURF collected extensive data, and therefore additional detailed information about participants for the present project will be available. C-SURF also provides an up-to-date address registry and a tracking team, which will be useful to keep drop-out rates low.

\section{Recruitment}

First, all French-speaking men involved in the C-SURF study on 25 September 2017 with a valid email address have been invited by email to complete a ten-question online version of the Alcohol Use Disorder Identification Test (AUDIT) (five min) ${ }^{27} 28$ and have been informed that they may be contacted for the whole study if they are selected within the following six months. A second email was sent two weeks later to the participants who did not answer the questionnaire.

Second, we will select participants using a random stratified sample selection. All the participants who complete the AUDIT and meet the inclusion criteria (see below) will be separated in two strata (AUDIT $\geq 13$; AUDIT $<13$ ), called groups hereafter. A total of 173 participants will be selected in the first group and 107 in the second group, using randomised numbers with the software R.

Selected participants will be contacted by phone by the psychologists to invite them to participate in the clinical assessment. An appointment at the CHUV will be scheduled if a participant agrees to participate. The whole procedure of recruitment is presented in figure 1.

\section{Procedure}

During assessment in the CHUV, participants will complete a computer-assisted questionnaire. Then, they will participate in the structured interview with a psychologist. Biological samples will be collected afterwards. The visit will take $90 \mathrm{~min}$ on average. The participants will be blinded to the group to which they belong. The interviewers will also be blinded to the participants' group. The participant will be given an oral feedback on their alcohol use after the interview and a written feedback at the end of the study.

\section{Inclusion/exclusion criteria}

This study is nested in the C-SURF study, of which the inclusion criteria were:

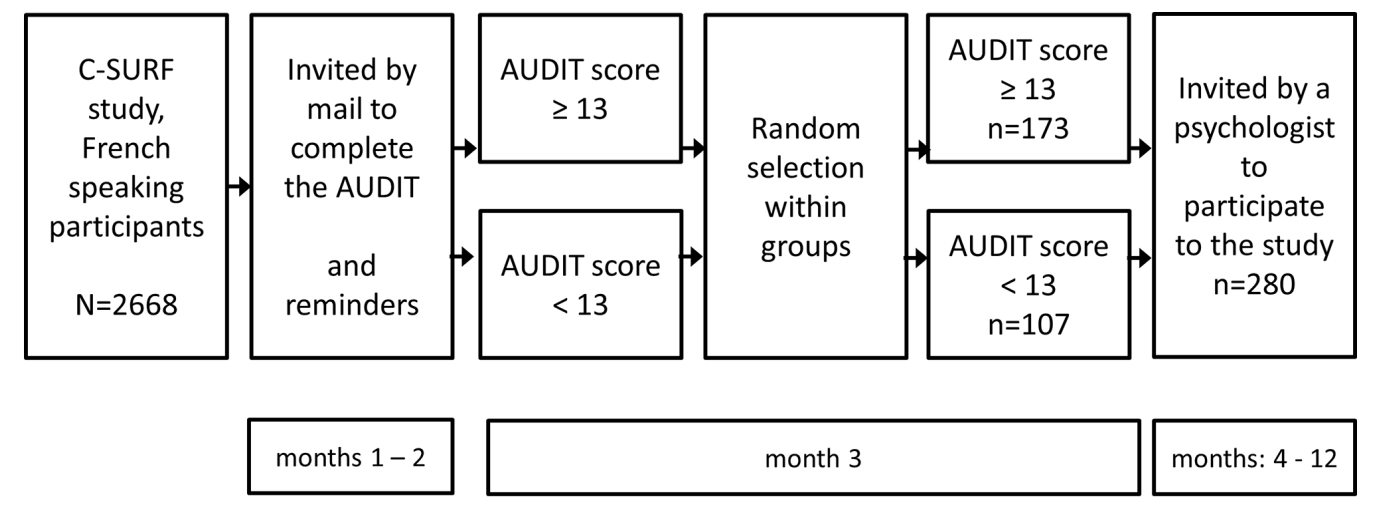

C-SURF: Cohort Study of Substance Use and Risk Factors; AUDIT: Alcohol Use Disorder Identification Test.

Figure 1 Flow diagram and timetable for the selection of study participants. 
- All young Swiss men at the army recruitment centre of Lausanne.

- All French-speaking cantons of Switzerland are included.

Within the French-speaking participants of the C-SURF, participants of the present study will be eligible if:

- They have a valid email address.

- They completed the AUDIT.

- They are randomly selected for the study's participation.

The exclusion criteria of our study are the following:

- They do not provide an informed consent to participate in the study.

- They have a score of zero on the three first questions of the AUDIT questionnaire related to alcohol use during the previous twelve months.

Hypotheses and research questions

Primary outcomes

Hypothesis 1

Self-reported AUD is not a reliable measure of alcohol-related dependence.

\section{Hypothesis 2}

HAU is a reliable measure of alcohol-related dependence.

\section{Secondary outcomes}

We also aim to investigate important secondary questions related to AUD and drinking patterns, as follows.

\section{Research question 1}

This question is related to the pattern of alcohol use and its relationship with alcohol-related dependence. Risky single-occasion drinkers, drinkers who drink six or more drinks on a single occasion, are more likely to be classified with alcohol-related dependence than non-risky single-occasion drinkers. We hypothesise that for the same moderate level of alcohol use, risky single-occasion drinking (RSOD) will be associated with a higher level of alcohol-related dependence, since this drinking pattern has been described as harmful. ${ }^{28-31}$ For example, people who drink seven drinks on Friday and Saturday (total 14 drinks per week) will have a higher level of alcohol dependence than people who drink three drinks on four different days (total 14 drinks per week). This hypothesis applies for moderate drinking levels because those who drink heavily are probably risky single-occasion drinkers (eg, five drinks per day). We also hypothesise that RSOD will also be associated with increased self-reported AUD. ${ }^{32}$

\section{Research question 2}

The second question deals with cut-offs for the biomarkers of chronic excessive drinking. More investigations are needed in order to propose relevant cut-offs for (ethyl glucuronide $(\mathrm{EtG})$ ) in hair and PEth (phosphatidylethanol (PEth) ) in blood. Evidence is still needed to define unhealthy alcohol use for decision-making. We will test the diagnostic performance of EtG and PEth compared with the clinical interviews. We will also test whether EtG and PEth are potential measures of RSOD, which is a question that has not been yet at focus, even if RSOD is a common drinking pattern among young people.

\section{Research question 3}

Another transversal research question will be to investigate non-response bias. Non-response bias is a crucial issue in surveys focusing on substance use. Indeed, contrariwise to most of the studies in which information about non-respondents are generally unavailable, data about the population (ie, C-SURF participants) will be available (eg, self-reported alcohol use, AUD, alcohol-related consequences, non-alcohol-related consequences, and mental and physical health). Therefore, we will be able to estimate non-response bias and predictors of non-response among participants who will be contacted to participate in the present study.

\section{Endpoint}

Primary endpoints

1. Alcohol-related dependence: Alcohol-related dependence will be assessed using a clinical interview over a twelve-month period. This diagnosis, our gold standard, will be based on the Diagnostic Interview for Genetic Studies (DIGS). ${ }^{33}$ It enables a comprehensive assessment of alcohol-related dependence and generates reliable diagnoses. It allows for the assessment of a comprehensive psychiatric diagnosis of alcohol-related dependence based on DSM-5 criteria. Its semistructured format ensures homogeneity across patients and interviewers. We will add three questions related to craving. ${ }^{34}$ Craving was added in the DSM-5, and the DIGS is available only according to the DMS-IV definition of alcohol-related dependence.

2. AUD: We will measure AUD as defined in the DSM-5, with eleven criteria. ${ }^{10}$ We will use the cut-offs recommended in the DSM-5 to define presence or absence of AUD (ie, two criteria out of eleven criteria), and also a continuous scale of criteria (from 0 to 11 , with a sum score of the eleven criteria). Moreover, following Martin $e t a l,{ }^{24}$ a restricted definition of primary symptoms of AUD will be computed by summing the items related to internal dysfunction (six criteria). We will use a continuous scale of criteria, since no cut-off is available for this operationalisation.

3. Alcohol use over time: Alcohol use over time will be measured with an extended quantity-frequency $(\mathrm{QF})$ questionnaire. The extended QF questionnaire captures the variability in drinking habits better than with other instruments, ${ }^{35}$ providing separate information on weekends and weekdays over a period of time (twelve months in our study). The measures are converted into a total number of drinks per week by multiplying average frequency of drinking and quantity of drinking. In order to define HAU, we will test different cut-offs (eg, the traditional cut-offs of two and four drinks on average per day and empirical cutoffs). 
4. Number of drinks according to past-week diary: The number of drinks during the past week assessed for each day separately will be added to create a total number of drinks for the whole week. A short-term recall measure (seven-day diary) will ask for the number of drinks during the past week on each day separately. This measure allows testing whether participants drink every day and how many drinks per day they drink.

5. Retrospective alcohol use: We will also collect more information on alcohol use over time using retrospective questions for participants at ages 10-15, 20 and 25 using questions used in the European Investigation into Cancer and Nutrition study, which described the trends of self-reported past consumption of alcohol use. ${ }^{36}$ Retrospective alcohol use will be modified to create an average number of drinks per week at ages $10-15,20$ and 25 . This measure will provide information on alcohol use over time.

6. Biomarkers of chronic excessive drinking: We will use the EtG in hair and the PEth in whole capillary blood. Two locks of hair (alternatively arm/chest hair) will be collected to assess EtG, and a capillary blood sample on a dried blood spot will be taken to measure PEth. Both biomarkers will be analysed by liquid chromatography coupled to tandem mass spectrometry using ISOvalidated methods (ISO: International Organization for Standardization). EtG and PEth are two recent biomarkers that appear especially reliable, ${ }^{9} 3738$ whereas traditional biomarkers (carbohydrate-deficient transferring and gamma-glutamyltransferase) lack sensitivity and/or specificity, especially among young people showing a typical RSOD behaviour on weekends or special occasions. Hair EtG is efficient to detect alcohol abuse and cut-offs have been proposed for at-risk drinkers ( $>20 / 30 \mathrm{~g}$ of ethanol/day) and heavy drinkers ( $>60 \mathrm{~g}$ of ethanol/day). Its sensitivity and specificity are very high $(>95 \%)$. On the contrary, it is less reliable for low levels of alcohol use. By contrast, PEth is useful to detect low levels of alcohol use during the last 2-4 weeks. Indeed, PEth has demonstrated a very high specificity (theoretically 100\%).

\section{Secondary endpoints}

1. RSOD: RSOD is often measured with an ordinal scale (eg, 'no RSOD', 'less than monthly RSOD', 'monthly RSOD', 'weekly RSOD' and 'daily RSOD') and with a cut-off of five or six drinks on a single occasion. ${ }^{39}$ The current study will propose more precise operationalisation of RSOD (eg, number of drinks per occasion, duration of each occasion and continuous scale for number of occasions).

2. Health issues and illnesses: The Short-Form Health Survey ${ }^{40}$ will be included with its two subscales: the mental component summary (mental and social health) and physical component summary (physical health).

3. Consequences: Sixteen consequences already used in C-SURF, which are not explicitly substance related, ${ }^{41}$ will be selected from standard instruments. ${ }^{42-45}$
Two sum-scores of consequence-associated scores will be computed: the first for social consequences and the second for health consequences. In addition, alcohol-related consequences will be assessed as in the DSM-5.

4. Quality of life (QOL): WHO Quality of Life Instrument-BREF has been validated widely, and it was found to be reliable and valid for use among patients with alcohol-related dependence. ${ }^{46}$ There are 26 questions rated on a five-point scale composed of two general question of QOL and four dimensions: physical health (seven items), psychological health (six items), social relationships (three items) and environment (eight items). Each question was rated in reference to the last two weeks. A percentage rating within each domain is computed with scores ranging from 0 (lowest QOL) to 100 (highest QOL).

5. Life satisfaction: The Satisfaction With Life Scale (SWLS) will be use to assess life satisfaction. ${ }^{47} \mathrm{~A}$ mean score of the five questions of the SWLS will be computed.

\section{Other variables}

For the selection of participants, we will use the AUDIT. ${ }^{2627}$ The AUDIT is a ten-item screening measure for AUD ${ }^{48} 49$ developed by the WHO, which includes three questions on dependence, four questions on specific consequences of harmful alcohol use and three questions on hazardous alcohol use. It has been described as a reliable screening tool of AUD. ${ }^{50}$

We will also assess demographic variables: age, educational status, and professional status.

Based on the C-SURF data (three waves already collected and available), we will match information on demographics, health, and substance use.

\section{Ethical aspects and safety \\ Consent and risks}

All procedures performed in studies involving human participants will be in accordance with the ethical standards of the institutional and/or national research committee and with the 1964 Declaration of Helsinki and its later amendments or comparable ethical standards. There is no expected adverse event or side effect for participants. Informed consent will be obtained from all individual participants included in the study.

\section{Confidentiality of the data}

Data generation, transmission, storage and analysis of health-related personal data and the storage of biological samples within this project will strictly follow the current Swiss legal requirements for data protection and will be performed according to the Human Research Ordinance (HRO) Art 5. Data protection and confidentiality will be guaranteed.

\section{Patient and public involvement}

Patients and public were not involved. 


\title{
AUD sens: 0.5, HAU sens: 0.55, Alpha:0.05, Margin of equivalence: 0.1
}

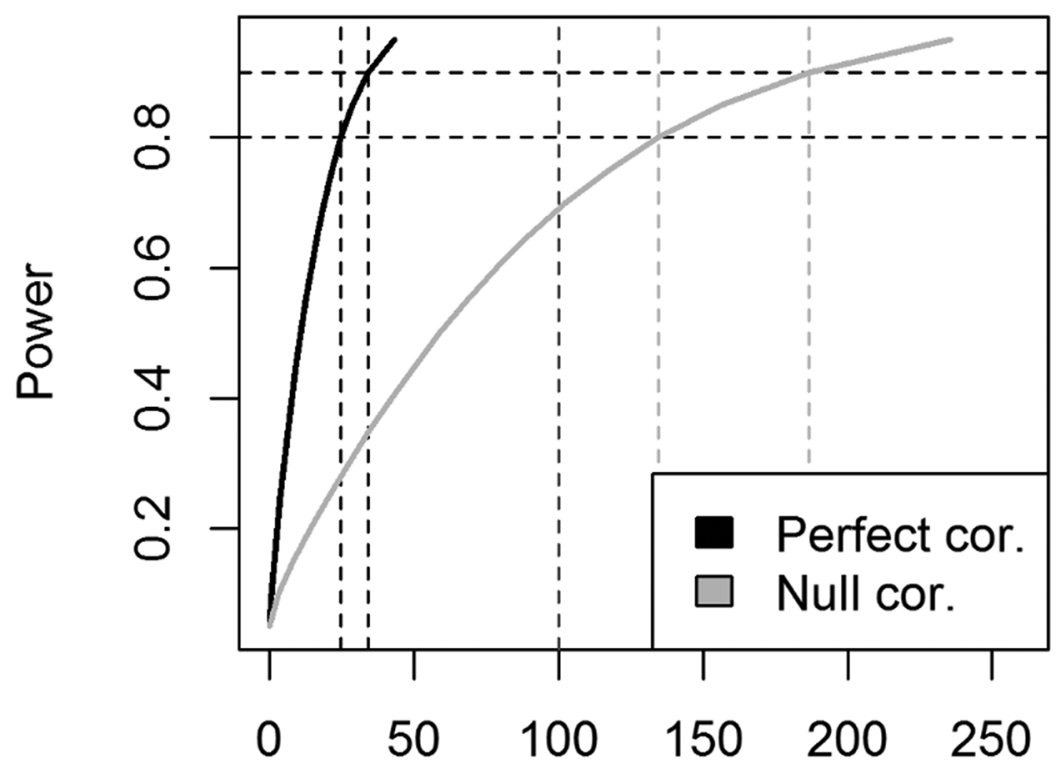

\section{Number of alcohol-related dependent patients}

\author{
(AUD sens: Alcohol use disorder sensitivity; HAU sens: Heavy alcohol use \\ sensitivity; cor: correlation)
}

Figure 2 Proportion non-inferiority test.

\section{Statistical analysis \\ Sample size}

There is no available information about the psychometric properties of the self-reported AUD nor of HAU. Therefore, it was not possible to estimate a precise sample size in a power calculation. To ensure that we have enough alcohol-related dependent participants to test the hypothesis of HAU being equivalent or better than self-reported AUD, we made several sample size calculations based on different scenarios of possible sensitivity of self-reported AUD (sensitivity between 0.2 and 0.8 ) using a proportion non-inferiority test with alpha $=5 \%$, a margin of equivalence of $10 \%$ and a difference in sensitivity between self-reported AUD and HAU of 5\%. ${ }^{51}$ The worst scenario is for sensitivity around $50 \%$ and no correlation between self-reported AUD and HAU. In this worst scenario, for a power of $80 \%, 135$ alcohol-related dependent participants are needed (as shown in figure 2). In a favourable scenario, with a power of $80 \%$ and a middle/large correlation (supported by the C-SURF data: $r=0.50$ ), a total of 67 participants with alcohol-related dependence are needed. We decided to choose a scenario between the worst and the most favourable with a correlation between self-reported AUD and HAU of 0.35, which is a moderate correlation between two related but different concepts. In this scenario, 86 participants with alcohol-related dependence are needed. Therefore, we will select at least
86 participants with alcohol-related dependence and 86 participants without alcohol-related dependence.

The AUDIT score will be used to select participants. Alcohol-related dependence is defined with a cut-off of 13 at AUDIT, ${ }^{52}$ with a sensitivity ranging between 0.78 and 0.90 and a specificity ranging between 0.87 and 0.92. ${ }^{50} 52$ The positive predictive values were estimated between 0.40 and $0.88 .^{50} 52$ Thus, by randomly selecting 151 participants with AUDIT greater or equal to 13 and a positive predicted value of 0.64 (midpoint between 0.40 and 0.88 ), there is a $95 \%$ probability of selecting at least 86 participants who are true positive. The negative predictive values were estimated at $0.97 .^{50} 52$ Therefore, we will select 93 participants with AUDIT lower than 13 in order to have a $95 \%$ probability of selecting at least 86 true negative non-alcohol-dependent participants. The psychologists will be blinded to the participants' AUDIT scores. In order to avoid issues related to attrition, we added $15 \%$ of participants in each group, a total of 173 participants with AUDIT $\geq 13$ and 107 participants with AUDIT $<13$ will be invited in each group $(n=280)$.

\section{Data analyses}

Analyses 1 (primary outcomes): HAU and AUD as measures of alcohol-related dependence

Considering the clinical interviews as a gold standard of alcohol-related dependence, and biomarkers as a gold 
standard of chronic excessive drinking, we will test the diagnostic performance of self-reported AUD and HAU measures to see whether they are suitable ways to assess alcohol-related dependence. We will use effect sizes to compare the correlations $\left(\mathrm{R}^{2}\right.$ to test common variance between measures and clinical effect size), and we will use Fisher's $\mathrm{R}$ to $\mathrm{Z}$ transformations to compare whether the correlations are significantly different from one another. Then, we will use dichotomised variables and test sensitivity, specificity, positive predicted value, and negative predicted value using the receiver operating characteristic (ROC) curves. To dichotomise alcohol use, different theory-oriented cut-offs will be compared (four, two and one drink(s) per day), and data-driven models will also be tested using stratum-specific likelihood ratio analysis and machine learning (random forests).

\section{Analyses 2 (primary and secondary outcomes): associations with health outcomes and alcohol-related variables}

We will compare outcomes' associations with the gold standards and the different self-reported measures (HAU, self-reported AUD and self-reported AUD without consequences). Effect sizes will be compared in order to know which measure is the best predictor of health and psychosocial issues and which one most resembles the associations with the gold standards.

\section{Analyses 3 (secondary research question): association of RSOD with alcohol-related dependence}

Associations of RSOD with the gold standard of alcohol-related dependence will be performed, adjusting for alcohol use (extended QF questionnaire) to assess its independent effect, and including an interaction between alcohol use and RSOD to investigate their combined effect.

\section{Analyses 4 (secondary research question): cut-off for biomarkers and associations with RSOD}

The diagnostic performance of EtG and PEth will be calculated for their optimal cut-off values selected with the ROC curves. Comparisons with clinical interviews, and HAU will be performed. We will also test whether EtG and PEth are potential measures of RSOD, using EtG and PEth cut-offs to predict RSOD using correlations and ROC curves. Different cut-offs will be tested in these analyses.

\section{Analyses 5 (secondary research question): non-response bias}

We will compare non-respondents to respondents using the information available in the previous waves of the C-SURF study using logistic regressions.

All analyses will use a two-sided $\alpha=0.05$. Statistical software will include SPSS V.24, Stata V.15 and R.

\section{DISCUSSION}

The main aim of this study is to test the quality of the self-reported AUD (also focusing on primary symptoms and excluding alcohol-related consequences) and of the self-reported HAU as measures of alcohol-related dependence as defined by the DSM-5 in a general population. The psychometric properties of the self-reported AUD and of the HAU will be tested against a clinical interview designed to diagnose alcohol-related dependence.

From an international perspective, the proposed project aims to address some methodological issues highlighted in recent studies related to the measure of substance-related dependence, and more specifically, alcohol-related dependence. The project will provide evidence regarding two important issues. First, it will test whether self-reported AUD, which is extensively used in alcohol research, is a reliable way to assess alcohol-related dependence. Second, it will investigate whether HAU is a reliable measure of alcohol-related dependence. Therefore, the study will provide insights on its capacity to capture alcohol-related dependence. The results of the study may have a large impact on future research on alcohol. It will suggest a better way to assess alcohol-related dependence in population-based samples and for screening perspectives. Additionally, the project will investigate thresholds needed for decision-making (early intervention and treatment), test the effect of drinking patterns on self-reported AUD, and determine cut-offs for biomarkers. These cut-offs will be useful for legal medicine, which needs further studies for decision-making regarding alcohol abstinence.

From a national perspective, this study will provide a valid prevalence rate of alcohol-related dependence among French-speaking young Swiss men in their mid-20s. It will be useful from a public health point of view. Moreover, cut-offs for unhealthy alcohol use will be proposed, which may be relevant for preventive purposes and may identify at-risk youths in Switzerland. It will improve screening for unhealthy alcohol use. It would also be useful for general practitioners to detect alcohol-related dependent persons. ${ }^{53}$

The current study is designed to provide evidence regarding the assessment of alcohol-related dependence in the general population and especially among young people. Potential benefits to society include a better understanding and evaluation of alcohol-related dependence, improvements of practices and future development of adequate public health planning. It will help to identify at-risk persons and groups. It may change the practices from a clinical and public health perspective, such as the use of alternative measures to screen for alcohol-related dependence and identify at-risk alcohol users, and from a research perspective, for example, to stop using unreliable self-reported addiction scales and thus to provide strong support to future findings in alcohol research.

\section{Author affiliations}

${ }^{1}$ School of Health Sciences (HEdS-FR), HES-SO University of Applied Sciences and Arts of Western Switzerland, Fribourg, Switzerland

${ }^{2}$ Center for the Understanding of Social Processes, University of Neuchâtel, Neuchâtel, Switzerland

${ }^{3}$ Forensic Toxicology and Chemistry Unit, Lausanne and Geneva Universities, Centre of Legal Medicine, Lausanne, Switzerland 
${ }^{4}$ Alcohol Treatment Centre, Lausanne University Hospital, Lausanne, Switzerland ${ }^{5}$ Centre for Addiction and Mental Health, Toronto, Ontario, Canada

${ }^{6}$ University of the West of England, Bristol, UK

${ }^{7}$ Division of Prison Health, Geneva University Hospitals and University of Geneva, Geneva, Switzerland

${ }^{8}$ Life Course and Social Inequality Research Center, University of Lausanne,

Lausanne, Switzerland

Acknowledgements The authors wish to thank Nicolas Fournier for his statistical support on sample size calculation and Stéphane Rothen for his insight on the DIGS and comments on the manuscript.

Contributors $\mathrm{KI}$ and SB wrote the manuscript. All authors critically reviewed the manuscript for important intellectual content. The study design and research proposal were mainly developed by SB and KI. GG, FS and J-BD made substantial contributions to the conception and the design of the study. The intervention was developed by SB and KI. All authors agreed to be accountable for all aspects of the work in ensuring that questions related to the accuracy or integrity of any part of the work are appropriately investigated and resolved. All authors read and approved the final manuscript.

Funding This study was funded by the Swiss National Science Foundation (no: 10001C_173418/1).

Competing interests None declared.

Patient consent Not required.

Ethics approval The protocol, information letters, questionnaires and the informed consent forms of the study have been approved by the Human Research Ethics Committee of the Canton of Vaud, Switzerland (no. 2017-00776).

Provenance and peer review Not commissioned; peer reviewed for ethical and funding approval prior to submission.

Open access This is an open access article distributed in accordance with the Creative Commons Attribution Non Commercial (CC BY-NC 4.0) license, which permits others to distribute, remix, adapt, build upon this work non-commercially, and license their derivative works on different terms, provided the original work is properly cited, appropriate credit is given, any changes made indicated, and the use is non-commercial. See: http://creativecommons.org/licenses/by-nc/4.0/.

\section{REFERENCES}

1. Rehm J, Anderson P, Barry J, et al. Prevalence of and potential influencing factors for alcohol dependence in Europe. Eur Addict Res 2015;21:6-18.

2. Rehm J, Marmet S, Anderson P, et al. Defining substance use disorders: do we really need more than heavy use? Alcohol Alcohol 2013;48:633-40

3. Room R. Alcohol and drug disorders in the international classification of diseases: a shifting kaleidoscope. Drug Alcohol Rev 1998;17:305-17.

4. Morse RM, Flavin DK. The definition of alcoholism. JAMA 1992;268:1012-4.

5. Rehm J. How should prevalence of alcohol use disorders be assessed globally? Int J Methods Psychiatr Res 2016;25:79-85.

6. WHO. ICD-10 classification of mental and behavioral disorder: clinical descriptions and diagnostics guidelines. Geneva: Switzerland: World Health Organization, 1992

7. American Psychiatric Association. Diagnostic and statistical manual of mental disorders. 4th Edn. Washington, DC: American Psychiatric Association, 2000.

8. Saunders J. What is this thing called addiction? Victoria, BC, 2014

9. Viel G, Boscolo-Berto R, Cecchetto G, et al. Phosphatidylethanol in blood as a marker of chronic alcohol use: a systematic review and meta-analysis. Int J Mol Sci 2012;13:14788-812.

10. American Psychatric Association. Diagnostic and statistical manual of mental disorders. 5th ed. Arlington, VA: American Psychiatric Publishing, 2013.

11. Baggio S, Studer J, Dupuis M, et al. Subthreshold problem drinkers in DSM-5 alcohol use disorder classification. Am J Addict 2016;25:408-15

12. Karriker-Jaffe KJ, Witbrodt J, Greenfield TK. Refining measures of alcohol problems for general population surveys. Alcohol Clin Exp Res 2015;39:363-70.

13. Slade T, Teesson M, Mewton L, et al. Do young adults interpret the DSM diagnostic criteria for alcohol use disorders as intended? a cognitive interviewing study. Alcohol Clin Exp Res 2013;37:1001-7.
14. Wakefield JC, Schmitz MF. How many people have alcohol use disorders? Using the harmful dysfunction analysis to reconcile prevalence estimates in two community surveys. Front Psychiatry 2014;5.

15. Pabst A, Kraus L, Piontek D, et al. Age differences in diagnostic criteria of DSM-IV alcohol dependence among adults with similar drinking behaviour. Addiction 2012;107:331-8.

16. Studer J, Baggio S, Mohler-Kuo M, et al. Differential association of drinking motives with alcohol use on weekdays and weekends. Psychol Addict Behav 2014;28:651-8.

17. Maraz A, Király O, Demetrovics Z. Commentary on: Are we overpathologizing everyday life? A tenable blueprint for behavioral addiction research. The diagnostic pitfalls of surveys: If you score positive on a test of addiction, you still have a good chance not to be addicted. J Behav Addict 2015;4:151-4.

18. Nutt DJ, Rehm J. Doing it by numbers: a simple approach to reducing the harms of alcohol. J Psychopharmacol 2014;28:3-7.

19. Saha TD, Stinson FS, Grant BF. The role of alcohol consumption in future classifications of alcohol use disorders. Drug Alcohol Depend 2007;89:82-92

20. Kandel DB. Drug and drinking behavior among youth. Annu Rev Sociol 1980;6:235-85.

21. O'Grady MA. Alcohol self-presentation: the role of impression motivation and impression construction. J Appl Soc Psychol 2013;43:854-69.

22. Department of Health. Alcohol guidelines review - Report from the Guidelines development group to the UK chief medical officers. London, UK: Department of Health, 2016.

23. Demetrovics $Z$, Király $O$. Internet/gaming addiction is more than heavy use over time. Addiction 2016;111:523-4.

24. Martin CS, Langenbucher JW, Chung T, et al. Truth or consequences in the diagnosis of substance use disorders. Addiction 2014;109:1773-8.

25. Gmel G, Akre C, Astudillo M, et al. The Swiss cohort study on substance use risk factors - findings of two waves. Sucht 2015;61:251-62.

26. Kraus L, Augustin R. Measuring alcohol consumption and alcoholrelated problems: comparison of responses from self-administered questionnaires and telephone interviews. Addiction 2001;96:459-71.

27. Bohn MJ, Babor TF, Kranzler HR. The Alcohol Use Disorders Identification Test (AUDIT): validation of a screening instrument for use in medical settings. J Stud Alcohol 1995;56:423-32.

28. Knight JR, Wechsler H, Kuo M, et al. Alcohol abuse and dependence among U.S. college students. J Stud Alcohol 2002;63:263-70.

29. Enoch MA, Goldman D. Problem drinking and alcoholism: diagnosis and treatment. Am Fam Physician 2002;65:441-8.

30. O'Connell J, Novins DK, Beals J, et al. The relationship between patterns of alcohol use and mental and physical health disorders in two American Indian populations. Addiction 2006;101:69-83.

31. Robin RW, Long JC, Rasmussen JK, et al. Relationship of binge drinking to alcohol dependence, other psychiatric disorders, and behavioral problems in an American Indian tribe. Alcohol Clin Exp Res 1998;22:518-23.

32. Baggio S, Dupuis M, Iglesias K, et al. Independent and combined associations of risky single-occasion drinking and drinking volume with alcohol use disorder: Evidence from a sample of young Swiss men. Drug Alcohol Depend 2015;154:260-3.

33. Berney A, Preisig M, Matthey ML, et al. Diagnostic interview for genetic studies (DIGS): inter-rater and test-retest reliability of alcohol and drug diagnoses. Drug Alcohol Depend 2002;65:149-58.

34. Ansseau M, Besson J, Lejoyeux M, et al. A French translation of the obsessive-compulsive drinking scale for craving in alcoholdependent patients: a validation study in Belgium, France, and Switzerland. Eur Addict Res 2000;6:51-6.

35. Gmel G, Studer J, Deline S, et al. More is not always bettercomparison of three instruments measuring volume of drinking in a sample of young men and their association with consequences. J Stud Alcohol Drugs 2014;75:880-8.

36. Klipstein-Grobusch K, Slimani N, Krogh V, et al. Trends in selfreported past alcohol intake from 1950 to 1995 observed in eight European countries participating in the European Investigation into Cancer and Nutrition (EPIC) project. IARC Sci Publ 2002;156:169-72.

37. Isaksson A, Walther L, Hansson T, et al. Phosphatidylethanol in blood (B-PEth): a marker for alcohol use and abuse. Drug Test Anal 2011;3:195-200.

38. Kharbouche H, Faouzi M, Sanchez N, et al. Diagnostic performance of ethyl glucuronide in hair for the investigation of alcohol drinking behavior: a comparison with traditional biomarkers. Int J Legal Med 2012;126:243-50. 
39. Gmel G, Kuntsche E, Rehm J. Risky single-occasion drinking: bingeing is not bingeing: Risky single-occasion drinking. Addiction 2011;106:1037-45.

40. Ware J, Kosinski M, Keller SD. A 12-Item Short-Form Health Survey: construction of scales and preliminary tests of reliability and validity. Med Care 1996;34:220-33.

41. Gmel G, Labhart F, Fallu JS, et al. The association between drinking motives and alcohol-related consequences - room for biases and measurement issues? Addiction 2012;107:1580-9.

42. Bucholz KK, Cadoret R, Cloninger $\mathrm{CR}$, et al. A new, semi-structured psychiatric interview for use in genetic linkage studies: a report on the reliability of the SSAGA. J Stud Alcohol 1994;55:149-58.

43. Hesselbrock M, Easton $\mathrm{C}$, Bucholz KK, et al. A validity study of the SSAGA-a comparison with the SCAN. Addiction 1999;94:1361-70.

44. Hibell B, Guttermsson U, Ahlström S, et al. The 2011 ESPAD report - Substance use among students in 36 European countries. Stockholm, 2012.

45. Wechsler H, Davenport A, Dowdall G, et al. Health and behavioral consequences of binge drinking in college. A national survey of students at 140 campuses. JAMA 1994;272:1672-7.

46. da Silva Lima AF, Fleck M, Pechansky F, et al. Psychometric properties of the World Health Organization quality of life instrument (WHOQoL-BREF) in alcoholic males: a pilot study. Qual Life Res 2005;14:473-8.
47. Diener E, Emmons RA, Larsen RJ, et al. The Satisfaction With Life Scale. J Pers Assess 1985;49:71-5.

48. Gache P, Michaud P, Landry U, et al. The Alcohol Use Disorders Identification Test (AUDIT) as a screening tool for excessive drinking in primary care: reliability and validity of a French version. Alcohol Clin Exp Res 2005;29:2001-7.

49. Saunders JB, Aasland OG, Babor TF, et al. Development of the Alcohol Use Disorders Identification Test (AUDIT): WHO Collaborative project on early detection of persons with harmful alcohol consumption-II. Addiction 1993;88:791-804.

50. Lundin A, Hallgren M, Balliu N, et al. The use of alcohol use disorders identification test (AUDIT) in detecting alcohol use disorder and risk drinking in the general population: validation of AUDIT using schedules for clinical assessment in neuropsychiatry. Alcohol Clin Exp Res 2015;39:158-65.

51. Zhou X-H, Obuchowski NA, McClish DK. Statistical Methods in Diagnostic Medicine. 2nd Edn. Hoboken, N.J: Wiley-Blackwell, 2011.

52. Meneses-Gaya C, Zuardi AW, Loureiro SR, et al. Is the full version of the AUDIT really necessary? Study of the validity and internal construct of its abbreviated versions. Alcohol Clin Exp Res 2010;34:1417-24.

53. Rehm J, Allamani A, Della Vedova R, et al. General practitioners recognizing alcohol dependence: a large cross-sectional study in 6 European countries. Ann Fam Med 2015;13:28-32. 
Correction: Comparison of self-reported measures of alcoholrelated dependence among young Swiss men: a study protocol for a cross-sectional controlled sample

Iglesias K, Sporkert F, Daeppen J, et al. Comparison of self-reported measures of alcoholrelated dependence among young Swiss men: a study protocol for a cross-sectional controlled sample. BMJ Open 2018;8:e023632. doi: 10.1136/bmjopen-2018-023632

This article was previously published with an error.

Reference 16 "Studer J, Baggio S, Mohler-Kuo M, et al. Differential association of drinking motives with alcohol use on weekdays and weekends. Psychol Addict Behav 2014;28:651-8." was incorrect. The correct reference is:

Baggio S, Iglesias K, Studer J, et al. Is the relationship between major depressive disorder and self-reported alcohol use disorder an artificial one? Alcohol and Alcoholism 2015; 50:195-199.

Open access This is an open access article distributed in accordance with the Creative Commons Attribution Non Commercial (CC BY-NC 4.0) license, which permits others to distribute, remix, adapt, build upon this work non-commercially, and license their derivative works on different terms, provided the original work is properly cited, appropriate credit is given, any changes made indicated, and the use is non-commercial. See: http://creativecommons.org/licenses/by-nc/4.0/.

(C) Author(s) (or their employer(s)) 2019. Re-use permitted under CC BY-NC. No commercial re-use. See rights and permissions. Published by BMJ.

BMJ Open 2019;9:e023632corr1. doi:10.1136/bmjopen-2018-023632corr1

A) Check for updates 